ORIGINAL ARTICLE

\title{
The importance of irradiance and area in neonatal phototherapy
}

G Hart, R Cameron

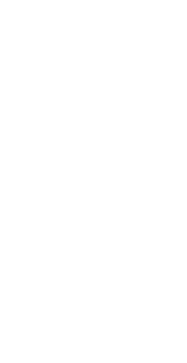

Arch Dis Child Fetal Neonatal Ed 2005;90:F437-F440. doi: 10.1136/adc.2004.068015

See end of article for authors' affiliations

Correspondence to:

Mr Hart, Medical Physics

Department, Bradford

Royal Infirmary,

Duckworth Lane, Bradford

BD9 6RJ, UK;

graham_hart_1953@

yahoo.com

Accepted 2 March 2005

Published Online First

4 May 2005 Background: Despite a long period of development, there are still considerable variations in the spectral
output, the levels of irradiance, and irradiated area provided by commercial phototherapy systems. These variations depend on the types and output of the lamps used to produce the phototherapy, along with the design of the systems, and principally on whether the phototherapy is provided from overhead or underneath.

Objective: To see whether commercially available phototherapy systems produce sufficient irradiance over the surface area of the neonate.

Methods: Surface plots of the output irradiance were made on a number of systems and used to calculate the effective irradiance on the surface of a premature or term baby, using mapped outlines.

Results: A 10-fold difference in peak central irradiances was found between the systems tested, with a fourfold to fivefold difference in effective irradiance to the baby surfaces. Although work published over 20 years ago showed that levels of irradiance should reach $2 \mathrm{~mW} / \mathrm{cm}^{2}$ to achieve optimal effectiveness, some of the commercial systems tested do not appear to achieve this level.

Conclusion: Purchasers of neonatal phototherapy systems need to take into account whether the systems will produce sufficient irradiance over the area to ensure maximal effect, to keep the treatment time to a minimum.

A recent report from the American Academy of Pediatrics on neonatal hyperbilirubinaemia confirms the efficacy of phototherapy as a treatment. ${ }^{1}$ Despite the long period of development since the original paper of Cremer et $a l^{2}$ there are still considerable variations in the spectral output, levels of irradiance, and irradiated area provided by commercial phototherapy systems. This was noted in the American Academy of Pediatrics report.

It is clear that the irradiance produced by phototherapy systems is an important factor. Many authors have shown that increasing irradiance produces a faster rate of fall of serum bilirubin. ${ }^{3-8} \operatorname{Tan}^{9}$ showed over 20 years ago that irradiances of $2 \mathrm{~mW} / \mathrm{cm}^{2}\left(\sim 40 \mu \mathrm{W} / \mathrm{cm}^{2} / \mathrm{nm}\right)$ are needed before a possible plateau response in the rate of bilirubin degradation is reached. Nevertheless, some current commercial phototherapy systems do not produce these irradiances.

Bringing the lights closer could increase the irradiance of overhead phototherapy systems. As this cannot easily be achieved for neonates in incubators, one solution is to provide phototherapy from underneath, as the neonate thus lies closer to the lights. This has been shown to be more effective than conventional overhead phototherapy. ${ }^{10}$ Another solution is to provide phototherapy from both above and below. This also seems to show a greater level of efficacy than overhead phototherapy alone. ${ }^{11}$

The other parameter that affects the effectiveness of phototherapy is the area of the neonate irradiated by the system. Maisels ${ }^{8}$ highlighted the need to consider this issue, and it was also used by Dicken et al, ${ }^{12}$ Vreman et $a l^{14}$ and Wentworth ${ }^{14}$ in the assessment of the overall performance of phototherapy systems.

The above appear to confirm the idea that many conventional phototherapy systems do not produce sufficient irradiance to maximise the degradation of bilirubin.

If overhead phototherapy does indeed reach Tan's plateau value of optimum irradiance, logic suggests that the way to increase the effectiveness of phototherapy would be to effectively double the treatment area by irradiating the neonate from below as well as above.

\section{MATERIALS AND METHODS}

The effective irradiance of a number of phototherapy systems was assessed by taking a series of irradiance measurements at $2 \mathrm{~cm}$ intervals in the illuminated field. Measurements were made using an International Light IL1700 radiometer with an SED033 detector, a "BR" bilirubin filter, and a "W" cosine diffuser. The radiometer and detector were calibrated against a secondary standard spectroradiometer, using a Philips TL52 phototherapy lamp as the light source.

All measurements for the overhead phototherapy systems were made with a source to measurement distance of $45 \mathrm{~cm}$ at the centre of the field, representing a typical treatment distance for neonates in incubators. For the fixed or underneath systems, measurements were made on the surface of the treatment area for that system, which included a bluegreen gel mattress (JLJ Healthcare, Halesowen, West Midlands, UK). Measurements were made until the output had fallen to about $10 \%$ of the peak value or until the sides of the crib had been reached.

The method used for the irradiance mapping was similar to that used by Dicken et $a l^{12}$ and Vreman et al. ${ }^{13}$ The data were entered into Excel spreadsheets and then linearly interpolated from the $2 \mathrm{~cm}$ grid used during the measurement process into a $1 \mathrm{~cm}$ grid to aid the mapping process. Outlines of both a premature and a term baby, similar to that of Vreman et al, were then mapped on to other spreadsheets. The only significant difference from the approach of Vreman et al is the use of an ellipsoidal model to simulate the curved edges of the body. This model suggested that the effective irradiance within $1 \mathrm{~cm}$ of the edge of the head and body should be reduced from $100 \%$ to $80 \%$, and to $50 \%$ for the arms and legs, which reduces the overall effective irradiance by $\sim 14 \%$. Figure 1 shows the outline for the premature baby. 


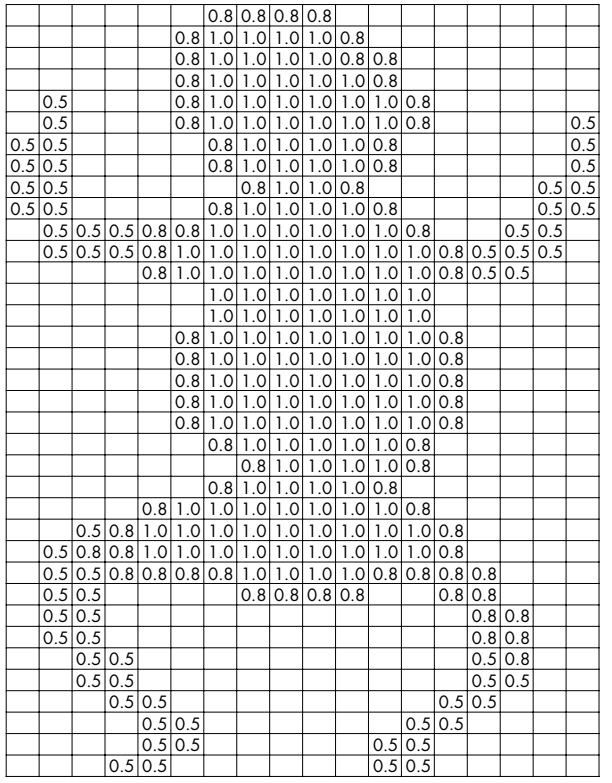

Figure 1 Premature baby outline, showing the weighting factors used in the calculation of total effective irradiance.
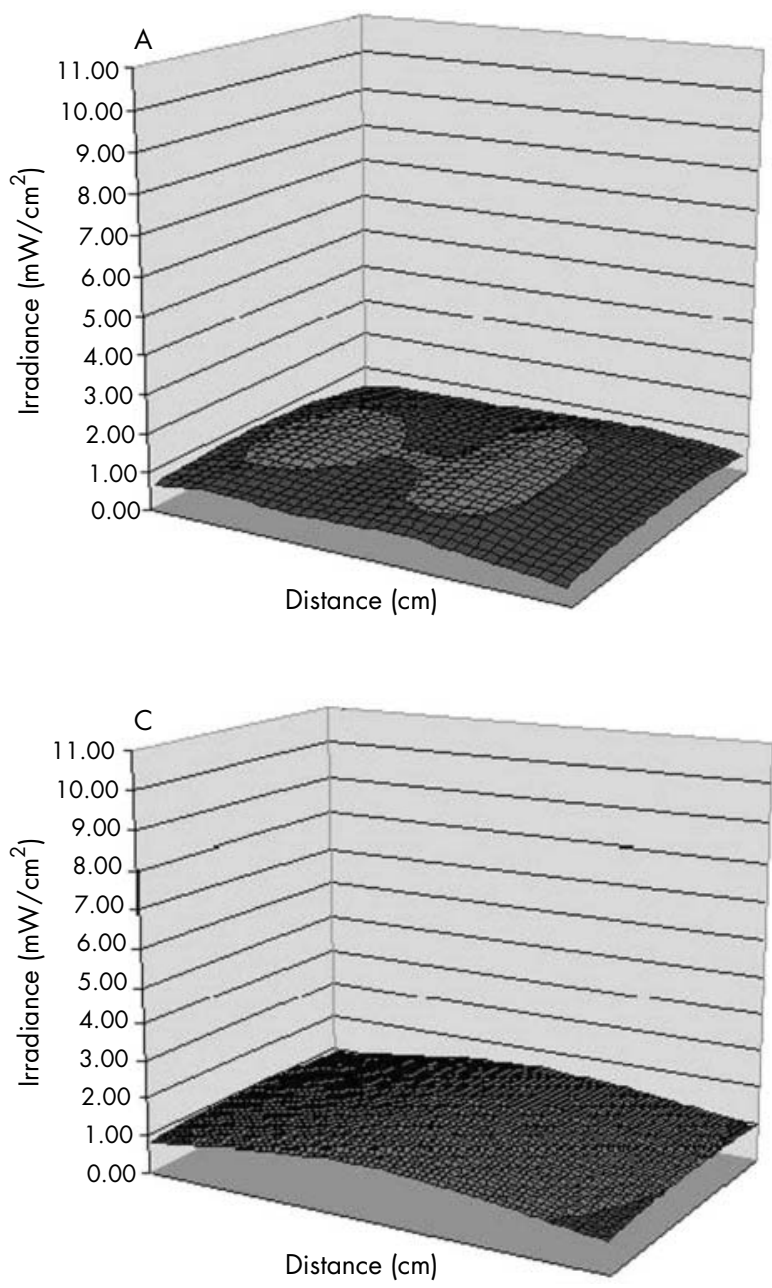

Each cell within the outlines was then multiplied by the corresponding interpolated irradiance values, centring the outlines of the premature and term babies at the centre of the irradiance fields. Values of the total irradiance were then calculated for the whole irradiated surface to assess the relative effectiveness of the phototherapy systems under test.

However, because neonates are known to move within the incubator or crib, the calculations were repeated with the outlines translated by $4 \mathrm{~cm}$ (and where possible $8 \mathrm{~cm}$ ) in both the $\mathrm{x}$ and $\mathrm{y}$ directions to assess whether this would make any difference to the received irradiance.

\section{RESULTS}

Measurements were made on a number of phototherapy systems:

- Hill-Rom Microlite, overhead unit based on white light sources;

- Draeger Phototherapie 800, overhead unit using a blue metal halide lamp;

- Medestime Duo, which has two independent overhead units, each containing four blue compact fluorescent lamps;

- Natus neoBLUE, overhead unit using a blue LED array (used here on its high output setting);
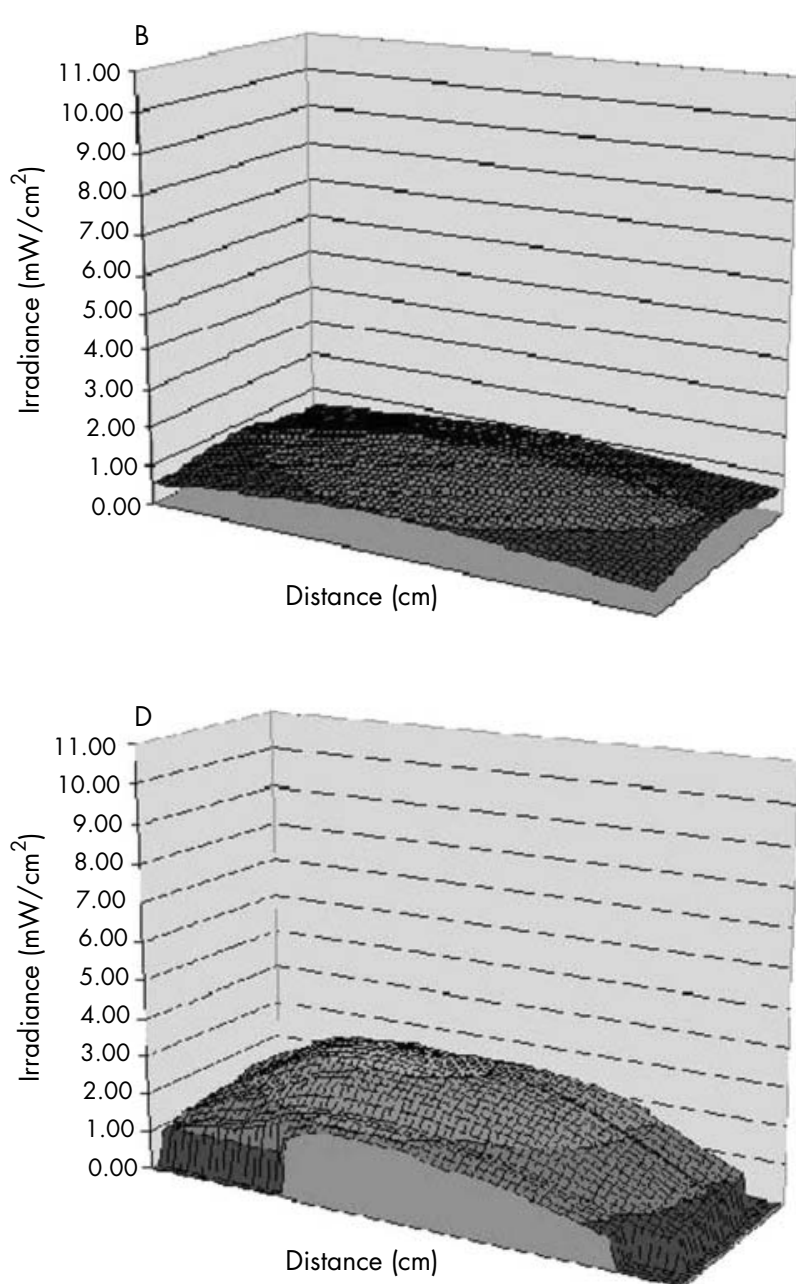

Figure 2 Surface plots for (A) Microlite, (B) Draeger, (C) Duo, and (D) neoBLUE phototherapy systems. The plots show a three dimensional representation of the irradiance produced by the phototherapy systems over the area of the crib. Irradiance on the vertical axis is measured in $\mathrm{mW} /$ $\mathrm{cm}^{2}$. The horizontal axes represent distance on the crib surface. 
A

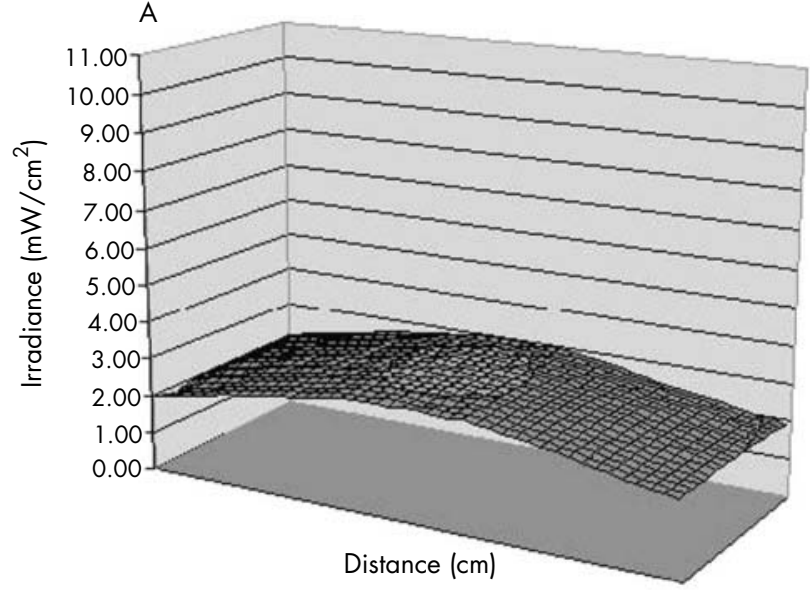

C

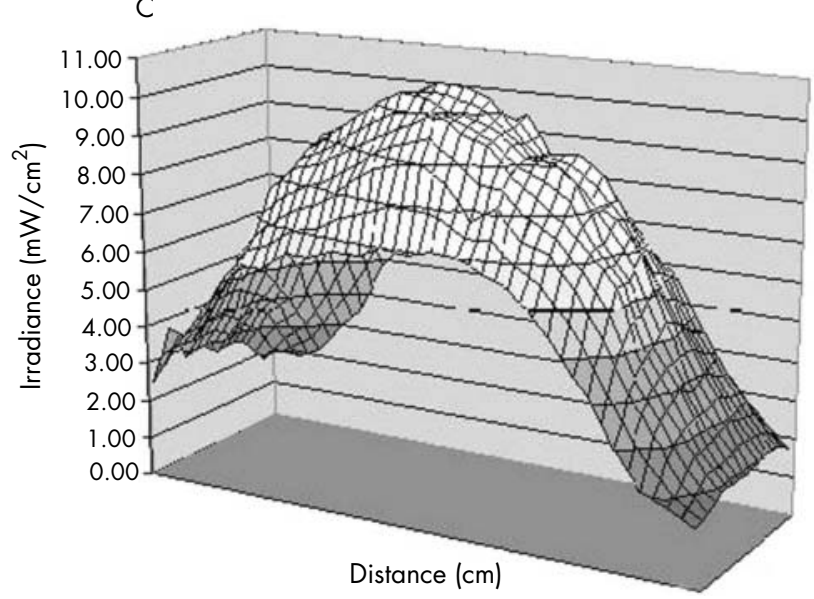

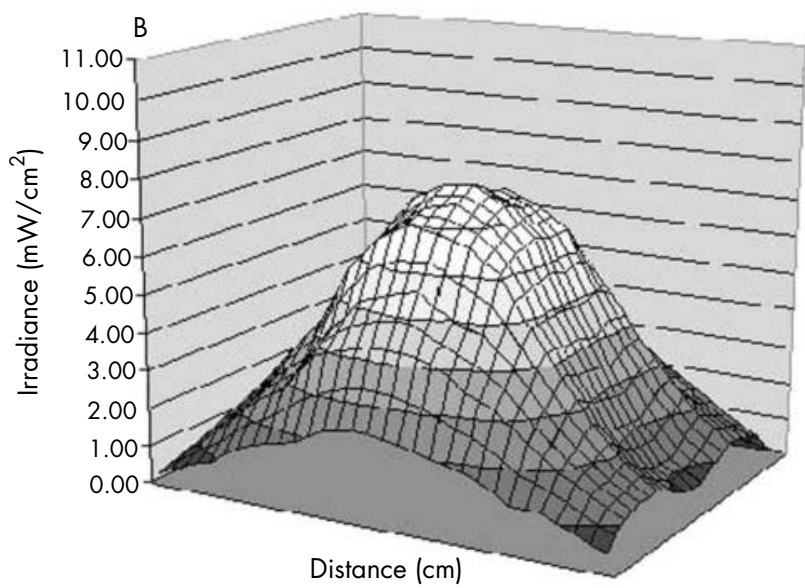

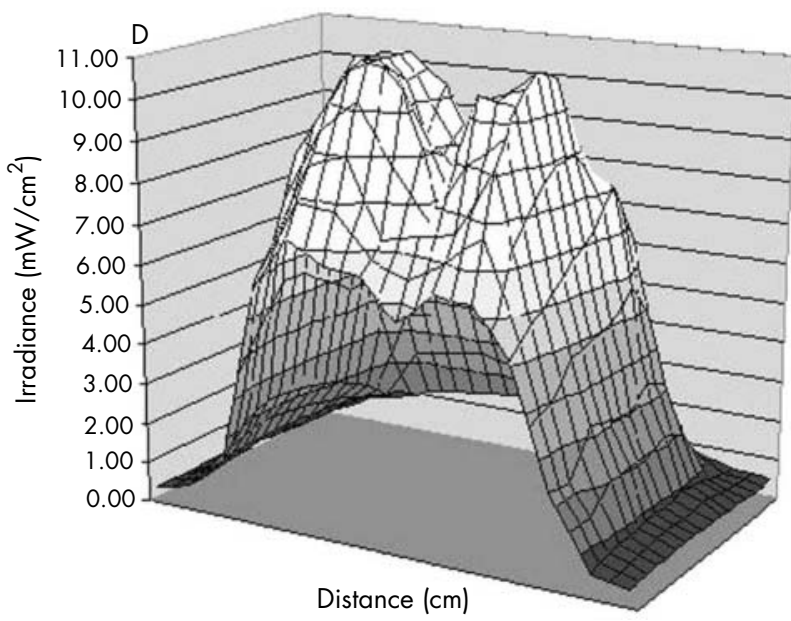

Figure 3 Surface plots for (A) IV2 (overhead), (B) Bilibed, (C) IV2 (under), and (D) LED phototherapy systems. The plots show a three dimensional representation of the irradiance produced by the phototherapy systems over the area of the crib. Irradiance on the vertical axis is measured in $\mathrm{mW} /$ $\mathrm{cm}^{2}$. The horizontal axes represent distance on the crib surface.

- Medestime Bilicrystal IV2, a dual phototherapy system based on six blue compact fluorescent lamps in an underneath unit, with a further five compact fluorescent lamps in a fixed overhead unit;

- Medela Bilibed, using a single blue compact fluorescent lamp underneath, with the baby lying on a blue-green gel mattress;

- A Medela Bilibed, modified in-house at Bradford as an experimental unit. This unit contained $800 \times$ Nichia NSB500 blue LEDs as the light source, with the baby lying on a blue-green gel mattress.

Figures 2 and 3 show the surface plots of the different units tested. All surface plots are plotted on the same vertical scale to aid direct comparison of the systems.

Table 1 gives the results of the measurements and calculations of total surface irradiance for both term and premature neonates. The ratio figures were obtained by dividing the mean of the moved total irradiance figures by the central total irradiance.

\section{DISCUSSION}

The surface plots in figs 2 and 3 clearly show the large differences in measured irradiance of the systems tested. This is mirrored in the total irradiance figures given in table 1.

The plots appear to show that the systems with lower levels of irradiance have a more even irradiance field than the higher output systems. Although this is true in absolute terms, the lower irradiance systems also show a similar percentage fall between centre and edge.

Three of the systems tested did not reach Tan's $2 \mathrm{~mW} / \mathrm{cm}^{2}$ central irradiance figure and also delivered lower total effective irradiances, which implies that they will not be as clinically effective as units that produce a greater level of irradiance.

Bringing the overhead units closer to the surface of the neonate would undoubtedly increase the measured irradiance, as the irradiance from an extended source is approximately proportional to $1 /$ distance from the source. This is usually not an option for a neonate being treated in an incubator, and for neonates in cribs the level of heat generated by the lights also has to be taken into account.

The underneath systems show a significantly higher level of irradiance than the overhead systems, principally because of the closeness of the light sources to the baby's skin surface. In these systems, the light has in fact been attenuated by the use of the gel mattress, which scatters the light and reduces the irradiance at the surface of the neonate by about $30 \%$. Use of a less attenuating surface would lead to a further increase in the irradiance.

The surface plot for the experimental LED array (fig 3D) shows a bimodal irradiance distribution, caused by the layout of the LEDs in two square panels with a small central gap. The resolution of the reduced irradiance between the panels 
Table 1 Peak and integrated irradiances of the systems under test, together with the ratios of the total irradiance when the outlines were moved $4 \mathrm{~cm}$ and $8 \mathrm{~cm}$

\begin{tabular}{llllll}
\hline & $\begin{array}{l}\text { Central peak } \\
\text { irradiance } \\
\left(\mathbf{m W} / \mathbf{c m}^{2}\right)\end{array}$ & $\begin{array}{l}\text { Central peak } \\
\text { irradiance } \\
\left(\mu \mathrm{W} / \mathbf{c m}^{2} / \mathbf{n m}\right)\end{array}$ & $\begin{array}{l}\text { Term baby total } \\
\text { irradiance at } \\
\text { centre }(\mathbf{m W})\end{array}$ & $\begin{array}{l}\text { Prem baby total } \\
\text { irradiance at } \\
\text { centre }(\mathbf{m W})\end{array}$ & $\begin{array}{l}\text { Ratio } \\
\mathbf{4} \mathbf{~ c m : c e n t r e ~}\end{array}$ \\
\hline Hill-Rom Microlite & 1.20 & $\sim 24$ & 319 & 236 & 0.98 \\
Draeger Phototherapie & 1.70 & $\sim 34$ & 575 & 366 & 0.97 \\
Medestime Duo & 1.72 & $\sim 34$ & 628 & 394 & 0.99 \\
Natus neoBLUE & 3.26 & $\sim 65$ & 1118 & 711 & 0.97 \\
Bilicrystal IV2 (top unit) & 3.08 & $\sim 62$ & 1034 & 684 & 0.99 \\
Bilicrystal IV2 (under unit) & 10.57 & $\sim 211$ & 2744 & 2033 & 0.97 \\
Medela Bilibed & 7.82 & $\sim 156$ & 1156 & 953 & 0.97 \\
LED Bilibed & 11.72 & $\sim 234$ & 1333 & 1215 & \\
\hline
\end{tabular}

shows the sensitivity of the method to changes in the shape of the light field.

Although there is a clear relation between the central irradiance value and the overall total irradiance figures for the term and premature baby outlines, they do not correlate perfectly. This is because of differences in the shapes of the light fields, and shows that both the peak irradiance and the area irradiated must be considered when seeking to assess the overall likely clinical effectiveness of the system.

Moving the outline by either $4 \mathrm{~cm}$ or $8 \mathrm{~cm}$ over the irradiated field did not appear to lead to a significant change in the level of irradiance. This was not done for either the Medela Bilibed or the modified unit containing the LED array, as the units are designed so the baby remains in a fixed position relative to the lights.

The irradiance measurements here were all made with a fixed bandwidth broad band radiometer. Maisels ${ }^{8}$ reminds us that we have to be careful about "measuring" irradiance, and Costarino et $a l^{15}$ go further, saying that "information obtained from a fixed band width radiometer designed to measure radiant flux in the blue light range may not allow meaningful comparison of phototherapy units in the clinical setting". This problem occurs because of the widely differing spectra of phototherapy systems. Some authors have decided to use spectroradiometric measurements, such as Wentworth, ${ }^{14}$ who integrated the spectra from $400-550 \mathrm{~nm}$.

However, using this kind of technique means that wavelengths outside the usually accepted action spectrum will contribute to the "useful" measured irradiance. The BR filter attached to the detector in our measurements is set to allow wavelengths in a $50 \mathrm{~nm}$ band centred on $450 \mathrm{~nm}$, to match the action spectrum of Cremer et al. ${ }^{2}$ The comments of Costarino et al $^{15}$ assume that wavelengths outside the blue region make a positive contribution to phototherapy. If this is not the case, however, measurement devices such as the one used here do indeed provide a reasonable way of assessing the useful irradiance of phototherapy systems. Most evidence thus far appears to support the action spectrum of Cremer et al and so this was used.

Reviews such as those produced by the UK's Medicines and Healthcare Products Regulatory Agency highlight the value of comparing systems using the same measurement techniques and equipment. ${ }^{16}$ This paper also shows that direct comparisons can show significant differences between commercially available systems of both the central and total effective irradiance over the surface of the neonate.

Purchasers of neonatal phototherapy systems need to take into account whether the systems will produce sufficient irradiance over the area required to ensure that the phototherapy is maximally effective, as this is likely to reduce the time that the neonate needs to undergo the treatment.

\section{ACKNOWLEDGEMENTS}

We acknowledge Medela for providing two Bilibeds for use in these measurements. We are grateful for permission to modify one of the Bilibeds, which was converted to hold the blue LED array. We are, however, responsible for the design and performance of the experimental LED array. We also thank Jackie Lloyd-Jones of JLJ Healthcare for the loan of the Medestime phototherapy equipment. We are grateful to Hugh Morrison for making many of the measurements that formed the surface irradiance mapping, and to Marina Hart, Stephanie Wentworth, and Diane Crawford for their many helpful comments during the writing of the final script.

\section{Authors' affiliations}

G Hart, R Cameron, Medical Physics Department, Bradford Royal Infirmary, Bradford BD9 6RJ, UK

Funding: This project was funded through an NHS R\&D programme at Bradford Teaching Hospitals NHS Foundation Trust.

Competing interests: none declared

\section{REFERENCES}

1 Ip S, Chung M, Kulig J, et al. An evidence-based review of important issues concerning neonatal hyperbilirubinaemia. Pediatrics 2004;114:130-53.

2 Cremer RJ, Perryman PW, Richards DH. Influence of light on the hyperbilirubinaemia of infants. Lancet 1958;1:1094-7.

3 Ennever JF. Blue light, green light, white light: more light in the treatment of neonatal jaundice. Clin Perinatol 1990;17:467-81.

4 Modi N, Keay AJ. Phototherapy for neonatal hyperbilirubinaemia: the importance of dose. Arch Dis Child 1983;58:406-9.

5 Holtrop PC, Ruedisueli K, Maisels MJ. Double vs single phototherapy in low birth weight newborns. Paediatrics 1992;90:674-7.

6 Tan KL, Lim GC, Boey KW. Efficiency of "high-intensity" blue light and "standard" daylight phototherapy for non-haemolytic hyperbilirubinaemia. Acta Paediatr 1992;81:870-4.

7 Kang JH, Shankaran S. Double phototherapy with high irradiance compared with single phototherapy in neonates with hyperbilirubinaemia. Am J Perinat 1995; 12:178-80.

8 Maisels MJ. Why use homeopathic dose of phototherapy? Paediatrics 1996:98:283-7.

9 Tan KL. The pattern of bilirubin response to phototherapy for neonatal hyperbilirubinaemia. Paediatr Res 1982;16:670-4.

10 Garg AK, Prasad RS, Hifzi IA. A controlled trial of high-intensity double surface phototherapy on a fluid bed vs. conventional phototherapy in neonatal jaundice. Paediatrics 1995;95:914-16.

11 Pometta P, Rodono A, Distefano G, et al. Double phototherapy with Wallaby optic fibres versus conventional phototherapy: case reports. Paediatric Medicine \& Surgery 1997;19:187-91.

12 Dicken P, Grant $\amalg$, Jones S. An evaluation of the characteristics and performance of neonatal phototherapy equipment. Physiol Meas 2000:493-503

13 Vreman HJ, Wong RJ, Murdock JR, et al. In-vitro efficacy of an LED-based phototherapy device (neoBLUE ${ }^{T M}$ ) compared to traditional light sources. Society for Pediatric Research Meeting, 2003 (poster)

14 Wentworth S. Neonatal phototherapy: which device is best for your babies? A review of nine devices. UK Neonatal Association Meeting, 2002 (poster).

15 Costarino AT, Ennever JF, Baumgart S, et al. Effect of spectral distribution on isomerisation of bilirubin in-vivo. J Pediatr 1985;17:125-8.

16 Medical Devices Agency. Neonatal phototherapy: a review including evaluations of the Ohmeda Biliblanket Plus and Medela Bilibed. MDA Report 391,2000 